\title{
LA AGENDA RELIGIOSA-CONSERVADORA EN EL DISCURSO POLÍTICO DURANTE LA CAMPAÑA ELECTORAL EN COSTA RICA $(2013-2014)^{1}$
}

\section{THE RELIGIOUS-CONSERVATIVE AGENDA IN THE POLITICAL DISCOURSE DURING THE ELECTORAL CAMPAIGN IN COSTA RICA (2013-2014)}

\author{
José Andrés Díaz González*
}

RESUMEN

\begin{abstract}
El presente artículo analiza la agenda religiosa-conservadora en el discurso político durante la campaña electoral nacional en Costa Rica (2013-2014). Para ello se estudia lo establecido en los Programas de Gobierno de los partidos políticos que aspiran a la Presidencia de la República, así como lo expresado en los debates políticos televisados por los principales aspirantes a la Presidencia. Se concluye que la agenda religiosa-conservadora no jugó un papel predominante en el discurso político-electoral, sin embargo, sigue siendo importante como estrategia discursiva para tratar de captar votos y atacar a los oponentes políticos.
\end{abstract}

PALABRAS CLAVE: COSTA RICA * ELECCIONES * DISCURSO * POLÍTICA * CONSERVADURISMO * RELIGIÓN

\section{ABSTRACT}

This paper analyzes the religious-conservative agenda in the political discourse during the national electoral campaign in Costa Rica (2013-2014). To perform the analysis, ideas established by aspiring parties in their Government Programs were studied, as well as the statements that were offered by the main aspirants to the Presidency during televisive debates. It is concluded that the religious-conservative agenda did not play a predominant role in the political-electoral discourse, nevertheless, it seems to be still important as a discursive strategy for vote capture and political attack.

KEYWORDS: COSTA RICA * ELECTIONS * SPEECHES * POLITICS * CONSERVATISM * RELIGION

Escuela de Ciencias Políticas, Universidad de Costa Rica e Instituto de Estudios Sociales en Población, Universidad Nacional, Costa Rica.

joseandres.diaz@ucr.ac.cr so religioso en la campaña electoral 2013-2014" (Proyecto N. ${ }^{\circ}$ 213-B5-080), realizado en la Escuela de Ciencias Políticas de la Universidad de Costa Rica. El autor desea agradecer a Karla Acuña Salas y Rebeca Solano, asistentes de investigación del proyecto, su valiosa colaboración en el proceso de recolección de datos y fuentes necesarias para la elaboración del presente documento. Asimismo, el autor desea expresar su agradecimiento a la persona evaluadora por sus valiosas observaciones y comentarios que ayudaron a enriquecer el texto; sin embargo, cualquier error u omisión presente en el artículo es responsabilidad del autor. 


\section{INTRODUCCIÓN}

Entrada la segunda década del siglo xxI, Costa Rica continúa siendo un Estado confesional católico, según lo establece el art. 75 de la Constitución Política, lo cual denota el peso que ha tenido la Iglesia Católica en la definición de los procesos políticos en el país. Además, una encuesta realizada en el 2013 por el Centro de Investigaciones y Estudios Políticos ( СIEP) de la Universidad de Costa Rica (ucR) indica que el $73 \%$ de la población entrevistada se declara católica y un $57,4 \%$ de esta defiende que el país sea un Estado confesional, demostrando la influencia que mantiene la Iglesia y la religión católica en el país.

Si bien, la propia Constitución Política y el Código Electoral prohíben expresamente el uso de símbolos o invocaciones religiosas de cualquier tipo para influir a las personas en materia electoral (art. 55, Código Electoral), en la última década ha proliferado el uso de elementos religiosos en la política electoral. A pesar de esto, actualmente se pueden hallar en el país un número importante de partidos en cuyo nombre se denominan abiertamente como "cristianos" o que en sus estatutos, planes de gobierno y discursos, hacen alusión a "valores cristianos" o elementos religiosos. Como apunta Jean-Pierre Bastian, la existencia de partidos que se identifican o abiertamente se exponen como "partidos religiosos" es un fenómeno común en América Latina que ha proliferado a partir de la década de 1980 (Bastian, 1999).

En el caso específico de Costa Rica, se puede mencionar al Partido Unidad Social Cristiana (Pusc), el cual se funda en 1983, cuya ideología se fundamenta parcialmente en la doctrina social de la Iglesia Católica; a lo cual le siguió la fundación de partidos políticos "evangélicos” en el país, siendo los casos más emblemáticos, el Partido Alianza Nacional Cristiana (PANC), el Partido Renovación Costarricense (PRC), el Partido Restauración Nacional (pro) y el Partido Alianza Demócrata Cristiana (PADC). Estas agrupaciones políticas no solamente han participado en varios procesos electorales (salvo el PADC que es de reciente formación), sino incluso han obtenido escaños en la Asamblea Legislativa. La transgresión de este tipo de agrupaciones a las normas que prohíben el uso de símbolos e invocaciones religiosas para la realización de campaña política ha llegado al punto que el Tribunal Supremo de Elecciones (TSE) se ha visto obligado a sancionarlos abiertamente (Rodríguez, 2013), algo inusual en la dinámica política electoral costarricense.

Se debe anotar que, a pesar de ser Costa Rica un país donde históricamente la religión católica ha sido la predominante, desde las últimas décadas del siglo xx ha aumentado el número de personas que se declaran afín o seguidora de una Iglesia Cristiana No-Católica (Evangélicos, Luteranos, Mormones, etc.). Este fenómeno no es exclusivo del país, sino que es una tendencia que ocurre a lo largo de toda América Latina (Bastian, 2012 y 2013). De esta manera, autores como Brian Smith (1998) y Paul Freston (2001) se han dado a la tarea de estudiar cual ha sido la incidencia que han tenido los movimientos protestantes en la vida política latinoamericana. Smith (1998) determina que no hay evidencia de que el surgimiento de iglesias de denominación pentecostal en América Latina haya ocasionado el mismo impacto político y económico que en Inglaterra y los Estados Unidos, donde la visión religiosa protestante fue un fuerte estímulo para la profundización de las democracias y el fortalecimiento de los sistemas económicos de libre mercado. Al contrario, este autor sugiere que las iglesias pentecostales han venido a fortalecer una visión autoritaria y clientelista de la política en Latinoamérica.

Sobre este tema, para el caso de Costa Rica, Timothy Steigenga (2001) hizo un análisis comparativo de lo que denomina la "pentecostalización de la religión", con el caso de Guatemala; su propósito es determinar como los cambios en la idiosincrasia religiosa pueden afectar las preferencias políticas de la población. Steigenga encuentra que, tras controlar otras variables relevantes socioeconómicas, las afiliación religiosa de la población resulta un pobre predictor de sus preferencias políticas. Sin embargo, su análisis le permite llegar a dos importante señalamientos, el primero, mientras en Guatemala las creencias pentecostales de la población se relacionan con la inactividad 
política y el apoyo al autoritarismo, esto no ocurre en Costa Rica. En otras palabras, mientras en Guatemala las creencias religiosas de tradición pentecostal influyen directamente en el comportamiento $y$ actitud política de la población, en Costa Rica las creencias religiosas de la población no son tan influyentes en su posición política; al menos para inicios del siglo xxı. El segundo señalamiento de Steigenga es que para el caso de Costa Rica, los políticos son los que se han apropiado de elementos discursivos, símbolos y prácticas propias de las iglesias pentecostales, debido a que estos elementos se han popularizado entre la población cristiana (incluyendo los católicos), por lo cual, la clase política ha llegado a adaptarlos como una estrategia para facilitar su identificación con el electorado (Steigenga, 2001).

Siguiendo lo anterior, se puede observar como la influencia de la religión no ocurre únicamente en los partidos de corte religioso; durante la campaña electoral 2009-2010, la entonces candidata a la Presidencia por el Partido Liberación Nacional (PLN), Laura Chinchilla Miranda, y el candidato por el Partido Movimiento Libertario (мL), Otto Guevara, participaron en una "Caminata en Defensa de la Familia", promovida por grupos afines a la Iglesia Católica y cuyo objetivo era manifestar su oposición a la legalización de la unión de personas del mismo sexo y al aborto (Díaz, 2012).

Incluso, la influencia de la religión en el plano político transcendió la propia campaña electoral; así, una vez electa Presidenta de la República, Laura Chinchilla visitó la Basílica de los Ángeles para ser nombrada "hija predilecta" de la Virgen de los Ángeles. En la misma ceremonia ella anunció que las puertas de Casa Presidencial estarían siempre abiertas para dialogar con los Obispos y demás jerarquías de la Iglesia Católica; un gesto que anunció la cercanía de su administración a esta institución y sus intereses (Arguedas, 2010).

La campaña política 2013-2014 continuó con una dinámica muy similar, al punto que sin importar su posición ideológica, los principales candidatos a la Presidencia de la República: Otto Guevara (ML), Jhonny Araya (PLN), José María Villata (FA) y Luis Guillermo Solís (PAC), aludían constantemente los valores religiosos $y$ a la figura de Dios en sus discursos políticos; esto a pesar que ninguno se declaraba un devoto católico practicante (Murillo, 2014).

$\mathrm{Si}$ a lo anterior se le suma lo establecido por Steigenga (2001), quién desde finales del siglo xx observa una apropiación por parte de la clase política costarricense de las prácticas $y$ elementos religiosos (principalmente pentecostales pero sin implicar una exclusión de los católicos) para ganar legitimidad ante la población, esta claro que existe por parte de los políticos una apropiación de elementos religiosos en sus discursos y propuestas de campaña. Por lo anterior, es que el presente artículo se centra en analizar el contenido de los programas de gobierno elaborados por los partidos que contendían a la presidencia de la República durante la campaña 2013-2014; así como, los discursos expresados por los cinco candidatos con mayor intención de voto $y$ aquellos pertenecientes a las agrupaciones políticas que abiertamente se identifican como "cristianas", esto con el propósito de observar cuál fue el uso que estos hicieron de los elementos y valores religiosos, como una estrategia para tratar de captar el apoyo del electorado.

\section{MÉTODO DE ANÁLISIS}

En el presente artículo se entenderá discurso como "una práctica social [...] una forma de acción entre las personas que se articulan a partir del uso lingüístico contextualizado, ya sea oral o escrito" (Casimigilia y Tusón, 1999, p.91). Así, Teun van Dijk al referirse al discurso como práctica social manifiesta que: "[l]a utilización discursiva del lenguaje no consiste solamente en una serie ordenada de palabras, cláusulas, oraciones y proposiciones, sino también en secuencias de actos mutuamente relacionados" (Van Dijk, 2000, p.151).

Los discursos analizados provienen de dos corpus o conjuntos de discursos diferentes. En primer lugar, se recolectaron todos los programas de gobierno elaborados por los trece partidos políticos que presentaron un candidato a la Presidencia de la República. En segundo lugar, se recolectaron las participaciones de los cinco candidatos con mayor intención de voto, 
así como aquellos pertenecientes a agrupaciones políticas "cristianas", en dos debates televisados: uno organizado por el Instituto de Formación y Educación en Democracia (IFED) del TSE, realizado entre el 5 y el 6 de enero de 2014 , y el otro organizado por la empresa Teletica (Canal 7), el día 19 de enero de 2014. La elección de dichos debates se debe a que al ser televisados y organizados por entes con trayectoria o legitimidad en la organización de dichos eventos, se puede presumir que fueron observados por una gran cantidad de televidentes (Carballo, 2015, p.28); por lo cual sería de esperar que los candidatos que participaran en estos, utilizaran los discursos que consideraran más atractivos para los y las votantes.

Para el análisis de los discursos se recurrió a utilizar el llamado "análisis pragmático del discurso" el cual, a diferencia de otro tipo de análisis de discurso, pretende ir más allá del análisis semántico y de las estructuras lingüísticas de los textos, para así incursionar en su contextualización. El propósito es observar cómo el discurso cobra sentido en un espacio $y$ tiempo específicos, es decir, en el contexto de su génesis y aplicación. En este sentido, Wodak y Meyer (2003) plantean que:

Una explicación plenamente "crítica" del discurso requeriría por consiguiente una teorización y una descripción tanto de los procesos y las estructuras sociales que dan lugar a la producción de un texto como de las estructuras sociales $y$ los procesos en los cuales los individuos o los grupos, en tanto sujetos históricos, crean sentidos para su interacción con los textos (p.19).

A partir de lo anterior es posible identificar una importante implicación analítica que esa técnica tiene en la investigación: la necesidad de realizar una ubicación o contextualización de lo que se expone en los discursos, en relación a su entorno, en este caso particular, la coyuntura política presente durante el desarrollo de la campaña electoral costarricense 2013-2014. Por lo tanto, a este tipo de análisis de discurso no le interesa estudiar la lingüística o la semántica que componen los actos comunicativos, sino que su interés se centra en determinar el valor estratégico de un discurso en un contexto determinado (Martín, 2009). En este sentido, la intención del presente análisis es observar como los partidos políticos y los candidatos a la Presidencia de la República incorporan elementos de la agenda-política conservadora en su discurso político, con el propósito de hacer su propuesta más atractiva para el electorado; o bien, generar un ataque en busca de mermar el apoyo recibido por sus contrincantes.

Como diversas autoras han señalado, la influencia de la religión en la política costarricense se ve claramente reflejada en la "agenda conservadora", la cual se vincula principalmente a los derechos sexuales y reproductivos, así como al papel que las Iglesias y las organizaciones religiosas deben tener en la discusión de temas públicos (Arguedas, 2010; Fuentes, 2006). Asimismo, la Iglesia Católica costarricense trató de posicionar estos temas en la discusión electoral durante la campaña 2013-2014 (Díaz, 2015). Por tales razones, se utilizaron estos temas como un marco de contexto para identificar el uso de elementos religiosos en los discursos políticos analizados.

\section{LA AGENDA CONSERVADORA-RELIGIOSA EN LOS PROGRAMAS DE GOBIERNO}

Los programas de Gobierno son los documentos en los que los partidos políticos exponen sus objetivos, metas y planes ha realizar, en el caso de obtener los votos necesarios para llegar a ser electos. Si bien, no se puede estimar que las y los electores hayan revisado, si quiera de forma superficial, los programas de Gobierno como un insumo para decidir por cual agrupación política votar; si es posible asumir que estos documentos son un reflejo del pensamiento político de los partidos y principalmente, muestran aquellos temas que les parecen más pertinentes o importantes de resaltar en la agenda pública. De esta manera, se puede suponer que las ideas que los partidos presentan en estos documentos serán la base tanto de su campaña como del discurso que su candidato exprese en distintos espacios $y$ foros. Sin embargo, se reconoce que pueden existir variaciones, 
omisiones o cambios en el discurso del partido conforme avanza la campaña electoral.

Asimismo, tampoco hay garantía que las agrupaciones políticas cumplan o desarrollen lo indicado en sus programas de Gobierno, ya sea por una falta de intención de su parte para cumplir lo prometido en campaña, así como por factores coyunturales que podrían afectar su plan de gobierno original. No obstante, a pesar de lo que suele ser la opinión general, diversas investigaciones han mostrado que las personas dedicadas a la política suelen ser más consecuentes y consistentes entre su pensamiento, su discurso y sus acciones que la persona ciudadana promedio, esto debido a que tienen fuertes incentivos para mantener una imagen que demuestre coherencia ante el electorado (Woshinsky, 2008). Dado lo anterior, se puede asumir que las propuestas expresadas en los planes de gobierno no son simplemente parte de un discurso que busca ser atractivo para la consecución de votos, sino que existen fuertes intenciones por parte de las agrupaciones políticas de mantener su actuación lo más coherente posible a lo dispuesto en estos documentos en caso de llegar al gobierno.

Por lo tanto, el análisis de los planes de gobierno permite visualizar las prioridades de los partidos y sus creencias políticas, en este caso particular, los elementos, las creencias $y$ los intereses religiosos que afectan su propuesta. Es necesario indicar que estas propuestas, así como, las promesas de campaña realizadas por los partidos políticos durante la campaña presidencial 2013-2014, no tuvieron como temas prioritarios aquellos relacionados con la agenda religiosa-conservadora (la cantidad de menciones de estos es bastante limitada en los planes de gobierno) y más bien, se concentraron en temas como la educación, el ambiente y la política fiscal (Nicolás, Alvarado, Rodríguez y Abarca, 2015). Esta situación llama la atención, ya que a pesar de que los partidos parecieran no tener como prioridad en su agenda política, articular los temas de la agenda religiosa-conservadora, estos son señalados y utilizados por sus candidatos a la Presidencia de la República con bastante frecuencia.

La tabla 1 muestra la posición de los partidos políticos en sus planes de gobierno, sobre distintos temas que se han identificado como punto de conflicto en la agenda conservadorareligiosa impulsada por distintos actores en Costa Rica. Además, la tabla hace un recuento del número de dichos temas que son abordados por cada partido político en sus programas. Es necesario aclarar que los partidos pueden posicionarse a favor o en contra de estos aspectos, por lo que el hecho que se mencionen reiteradamente, no puede interpretarse como que la agrupación apoye los temas de la agenda conservadora-religiosa. Sin embargo, si se puede inferir que estos les parecen relevantes como para tener la necesidad de pronunciarse, ya sea a favor o en contra. 


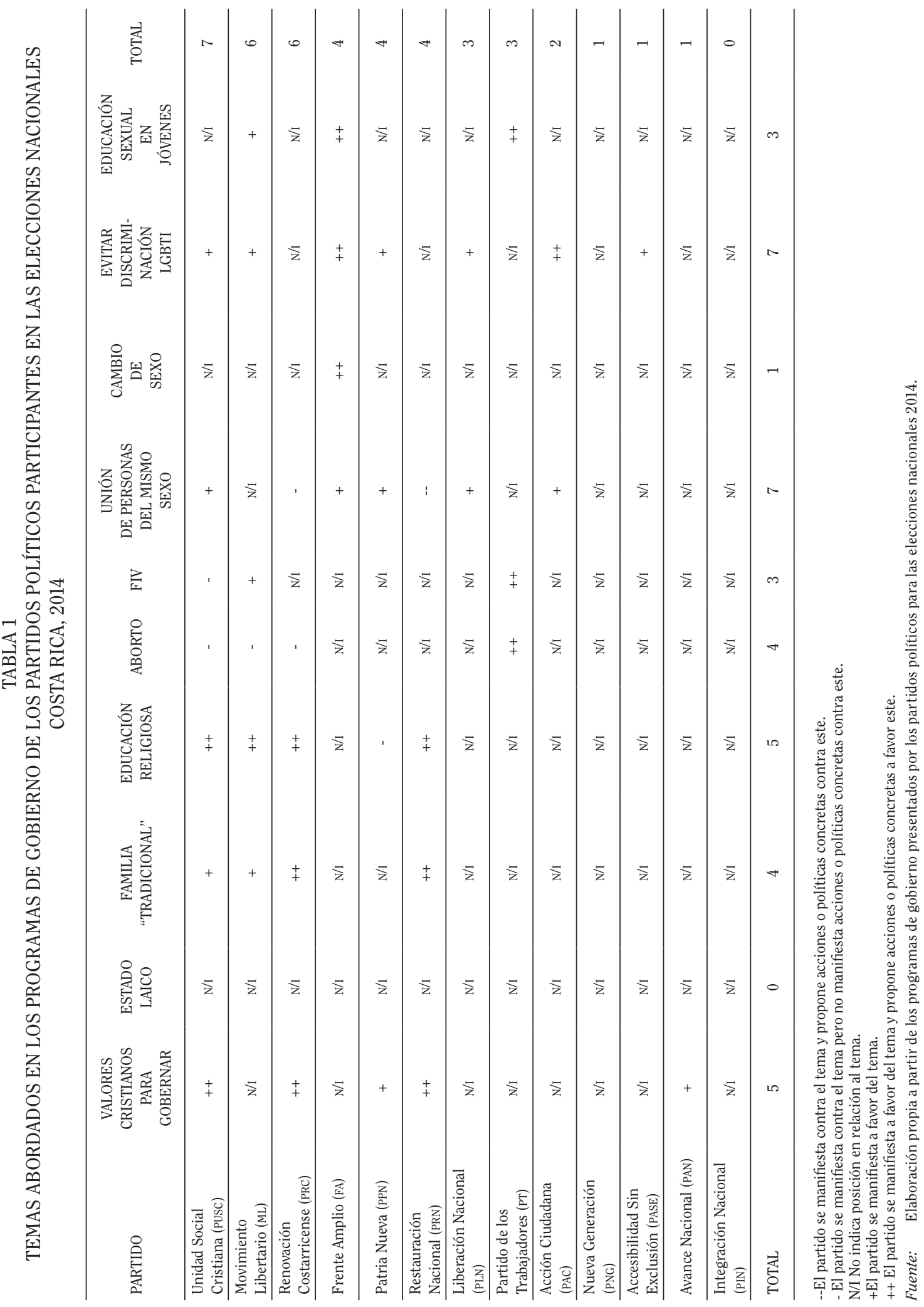

(c) (i) (-) Rev. Ciencias Sociales Universidad de Costa Rica, 157: 65-81 / 2017 (III). (ISSN: 0482-5276) 
El partido con mayor número de menciones en estos temas en su plan de gobierno es el pusc; aunque tiene posiciones "mixtas" sobre estos. Así, acoge temas que son congruentes con la agenda conservadora-religiosa, tales como: la defensa de la familia "tradicional", la promoción de la educación religiosa en el sistema educativo público, así como, una oposición al aborto y a la fiv. Sin embargo, al mismo tiempo, se manifiesta a favor de la legalización de las uniones de personas del mismo sexo y en la adopción de medidas para evitar la discriminación de la población Lesbiana, Gay, Bisexual, Transexual e Intersexual (LGвт).

Una situación similar ocurre con el $\mathrm{mL}$, el cual manifiesta en su plan de gobierno estar de acuerdo con temas de la agenda conservadora-religiosa (defensa de la familia tradicional, oposición al aborto, promoción de educación religiosa), pero al mismo tiempo indica estar a favor de evitar la discriminación de la población LGBTI y de promover la educación sexual en la población joven.

La situación varía al observar al PRc, si bien este partido menciona la misma cantidad de temas que el $\mathrm{mL}$, todos estos los aborda desde la promoción de la agenda conservadorareligiosa, lo cual es algo congruente al tratarse de un partido "cristiano". Eso también sucede con el PRN, el cual menciona una menor cantidad de temas respecto al pre, de una manera congruente con la agenda conservadora-religiosa.

Una mención especial merece el $\mathrm{FA}$, que es el partido que más temas menciona pero siempre opuesto a la agenda conservadorareligiosa. Así, esta agrupación política se manifiesta a favor de la legalización de la unión de personas del mismo sexo, la adopción de medidas para evitar la discriminación de la población LGBTI y la promoción de la educación sexual de personas jóvenes, además es la única agrupación política que toca el tema del cambio de sexo en personas transgénero, posicionándose a favor de este.

En cuanto a los temas abordados en los planes de gobierno, llama la atención que ninguna agrupación política toma posición respecto al Estado laico. Según el art.75 de la Constitución Política de Costa Rica, la religión oficial es la católica; debido a esto, diversos grupos ciudadanos e incluso, partidos políticos, han tratado de promover una reforma constitucional para eliminar que el Estado tenga religión oficial y por ende, se convierta en un Estado laico. Ante dicha situación, las altas autoridades de la Iglesia Católica junto a otras agrupaciones religiosas, han realizado una campaña para evitar que se "saque a Dios de la Constitución” (Díaz, 2015). Sin embargo, a diferencia de otros temas, esta posición de los grupos religiosos parece no hacer eco en los partidos políticos, los cuales no se manifiestan ni a favor ni en contra de un Estado laico, incluido los llamados partidos cristianos.

En cuanto a los temas, hay dos con mayor cantidad de menciones en los planes de gobierno: la posición (a favor o en contra) ante la legalización de las uniones de personas del mismo sexo y la adopción de medidas para evitar la discriminación de la población LGBTi. Ambas posiciones pueden considerarse relacionadas, ya que la legalización de las uniones entre personas del mismo sexo es una medida necesaria para disminuir la discriminación que vive parte de esta población. De esta manera, se puede observar que todas las agrupaciones políticas en cuyas propuestas se apoya, en alguna medida, la legalización de las uniones de parejas del mismo sexo, también manifiestan la necesidad de adoptar acciones para evitar la discriminación que sufre la población цGвті (ver tabla 1).

La legalización de las uniones de personas del mismo sexo es un tema que lleva varios años discutiéndose en la agenda pública $y$ donde claramente los opositores al tema han estado vinculados a grupos religiosos y fundamentalistas, inclusive, a la alta jerarquía de la Iglesia Católica costarricense (Díaz, 2012). Asimismo, a pesar que históricamente en Costa Rica hay una tendencia para invisibilizar $y$ discriminar abiertamente a este grupo por parte de la población e institucionalidad costarricense (Vargas, 2013); progresivamente la opinión pública ha tendido a cambiar su posición y a mostrarse favorable a permitir este tipo de uniones. Así, para el 2015, el 40,5\% de la población costarricense indica estar de acuerdo con 
la legalización de las uniones del mismo sexo (Programa Umbral Político, 2015).

Este cambio de tendencia también ha sucedido en la clase política costarricense; por ejemplo, para el 2012 la mayoría de los diputados del pase, los cuales habían mantenido una férrea oposición a cualquier proyecto de ley que tuviera como objetivo la legalización de las uniones de personas del mismo sexo, cambian su postura y se declaran a favor de dichas iniciativas, bajo el argumento de que son necesarias para garantizar el pleno disfrute de los derechos humanos de dicho grupo (Editor, 2012).

Tomado lo anterior en consideración, no es de extrañar que la mayoría de los partidos que abordan este tema en sus programas de gobierno lo hagan para indicar que se encuentran a favor de impulsar medidas para legalizar la unión de personas del mismo sexo; solamente dos partidos políticos se posicionan en contra: el PRN y el Prc. En el caso del PRc, no indica abiertamente que evitará la legalización de las uniones de personas del mismo sexo, pero señala que uno de sus principios doctrinarios es que la familia se basa únicamente en el matrimonio heterosexual, con lo que se da a entender que no reconocen otro tipo de uniones (Partido Renovación Costarricense, 2013).

Respecto al PRN, este adopta una posición similar al PRC en el sentido que no indica abiertamente que se opondrá a la legalización de las uniones entre personas del mismo sexo, sino que su visión de la familia solo se constituye mediante el matrimonio heterosexual. Sin embargo, va más allá, en el sentido que en su plan de gobierno expresa que es necesario "defender la unión heterosexual como la única y exclusiva manifestación de la institución del matrimonio" (Partido Restauración Nacional, 2013, p.7-8). Por lo tanto, al hacer referencia a la necesidad de "defender" la unión heterosexual, se puede inferir que para dicha agrupación hay un ataque contra esta, el cual proviene — posiblemente — de la posibilidad de legalizar las uniones entre personas del mismo sexo. En otras palabras, el PRN no solamente se opone a este tema porque sea contrario a sus convicciones $y$ creencias, sino también porque cree que aprobar la legalización de este tipo de uniones implicaría debilitar el matrimonio heterosexual.

Siguiendo lo anterior se encuentra el tema de la "defensa de la familia tradicional". Al respecto es necesario indicar que este es uno de los temas que impulsó la jerarquía de la Iglesia Católica costarricense para la campaña electoral 2013-2014, como parte de una estrategia discursiva para manifestar su oposición a la legalización de las uniones de personas del mismo sexo. Dado que, como se indicó anteriormente, la posición de la clase política y de la opinión pública costarricense ha ido variando con los años y es cada vez más proclive a favorecer los derechos de la población LGвті. El no oponerse abiertamente a esta puede concebirse como una estrategia de la Iglesia Católica para no generar el rechazo y enojo por parte de los grupos que se encuentran a favor; sin embargo, al defender que el único modelo válido, que debe ser reconocido social y legalmente es el de "familia tradicional" (matrimonio heterosexual), lo cual está evitando de manera indirecta que la población LGBTi obtenga los derechos necesarios para formar familias, ya que no se ajustan a dicha visión (Díaz, 2015).

De esta manera, no es de extrañar que el PRN $y$ el PRC también adopten un discurso similar sobre la necesidad de proteger a la "familia tradicional", el cual viene a servir como un apoyo a su postura de oposición a las uniones entre personas del mismo sexo. Sin embargo, los partidos que en su plan de gobierno indican estar de acuerdo en apoyar este último punto, al mismo tiempo indican en sus documentos que buscaran promover $y$ defender el modelo de "familia tradicional". Tal es el caso del pusc, el cual manifiesta constantemente en su plan de gobierno la necesidad de apoyar y fortalecer a las familias. En ningún momento hace referencia directa a la "familia tradicional", no obstante, al abordar el tema de los derechos de la población LGBTI indica que está a favor de garantizar sus derechos y promover acciones para combatir la discriminación, al mismo tiempo expresa que esto puede realizarse sin la necesidad de reformar el Código de Familia, es decir, sin que se acepte de manera legal que existen otros modelos de familia (Partido Unidad Social 
Cristiana, 2013). Por lo tanto, parece que existe cierta contradicción en la propuesta del pusc en lo que respecta a los derechos de la población LGBTI, ya que manifiesta estar a favor de estos $y$ procuraría en un eventual gobierno promover acciones para evitar la discriminación que sufre dicho grupo; no obstante, adopta una posición discriminatoria al no aceptar que estos puedan formar "familias".

Una situación similar ocurre con el ML, que en su plan de gobierno indica la necesidad de fortalecer $y$ promover los "valores familiares", aunque no aclara en que consisten estos, pero parece que los asocia con actitudes ligadas con las creencias religiosas de las personas (Partido Movimiento Libertario, 2013, p.55). Si a esto se le suma que no se pronuncia a favor de la legalización de las uniones de personas del mismo sexo, a pesar que - supuestamente- defiende la libertad de las personas como su máxima ideológica; es factible pensar que hace referencia a la promoción del modelo de familia tradicional.

Otro punto destacado es que cinco partidos manifiestan abiertamente que recurrirán a utilizar "valores cristianos", como elementos guías de las acciones a realizar en un eventual gobierno. En el caso de los partidos políticos evangélicos (el PRN y el PRC), no es de extrañar esta posición; incluso el programa de gobierno del PRN inicia exponiendo que su ideología es el "cristianismo social" (Partido Restauración Nacional, 2013). Tampoco es extraño esta postura en el caso del pusc, el cual abiertamente se declara como un partido socialcristiano, ideología que - al menos de manera nominal- ha mantenido desde su fundación en 1983 (Partido Unidad Social Cristiana, 2013).

Es importante resaltar la postura del PPN, una agrupación política que trata de posicionarse como una opción "progresista", pero que abiertamente indica que recurrirá a valores cristianos como base del accionar de un posible futuro gobierno, característica que comparte con las opciones más conservadoras de la oferta electoral costarricense. No obstante, se debe hacer la aclaración que el ppN manifiesta que recurrirá para dicho propósito a las encíclicas papales que hagan referencia a la reivindicación de las causas y problemas sociales, así como documentos similares provenientes de otras denominaciones del cristianismo (Rodríguez, 2013).

En cuanto al tema de la "educación religiosa", este hace referencia a las clases de religión (católica) que se imparten en las escuelas y colegios públicos de Costa Rica. Al respecto, el ML, el Pusc, el PRN y el PRC no solo indican en sus planes de gobierno que están a favor, sino que señalan la necesidad de fortalecerla (Partido Restauración Nacional, 2013; Partido Unidad Social Cristiana, 2013). El único partido que adopta una posición contraria es el ppN, el cual manifiesta la necesidad de reformar el sistema educativo del país, con el propósito que responda a "los principios de la laicidad, el humanismo, la pluralidad cultural, y la gratuidad" (Rodríguez, 2013, p.9). Un tema directamente vinculado es la educación sexual en centros educativos públicos, ya que históricamente, la Iglesia Católica ha buscado intervenir en la elaboración de las guías en esta materia, con el propósito de implantar su visión al respecto $y$ en años recientes, grupos evangélicos han protestado por la enseñanza de este tema en colegios públicos (Díaz, 2016; Quesada, 2012). A pesar de esto, los partidos políticos evangélicos o "cristianos" omiten manifestarse sobre este punto en su plan de gobierno y las tres agrupaciones políticas que sí lo mencionan (мL, ға y Рт), lo hacen para indicar la necesidad de apoyar o fortalecer este tipo de educación.

Respecto a los derechos reproductivos, se encuentran los casos de la fecundación in vitro (Fiv) y el aborto. En el año 2000, una resolución de la Sala Constitucional eliminó el reglamento que regulaba este procedimiento médico en el país, ante lo cual surgió un movimiento conformado por parejas que indicaban estar siendo perjudicadas por la imposibilidad del acceso a este; al mismo tiempo, distintos grupos políti$\cos y$ religiosos empezaron a realizar acciones para evitar que se permitiera nuevamente la fIv en Costa Rica. En el año 2013, un fallo de la Corte Interamericana de Derechos Humanos obliga a que en el país se vuelva a aplicar la técnica, sin embargo, no es hasta después de un segundo llamado realizado por este organismos 
internacional en el 2016, que el Estado costarricense permite la realización de la fiv. Esta temática es abordada por tres partidos, en el caso del pusc, que es el único partido que manifiesta alguna reticencia al tema, dice compartir la tesis original de la Sala Constitucional que prohíbe la realización de esta técnica en el país; no obstante, declara que dada "la resolución de la Corte Interamericana, debe legislarse la FIv pero para garantizar el derecho a la vida, como un instrumento para crear vida..." (Partido Unidad Social Cristiana, 2013, p.61). En cuanto a las agrupaciones políticas que mencionan estar abiertamente a favor de la frv están el mL y el PT, este último incluso indica la necesidad del acceso gratuito a esta técnica.

Por otra parte, en cuanto al tema del aborto es necesario mencionar que el art.121 del Código Penal, el único tipo de aborto que no se encuentra penalizado es el aborto terapéutico. Este es practicado cuando la vida de la mujer embarazada se encuentra en riesgo $y / 0$ en el caso de embarazos incompatibles con la vida extrauterina; ambos con el consentimiento de la mujer. En ese sentido, otras causales que podrían influir en una mujer para que decida realizar un aborto se encuentran prohibidas; es decir, cuentan con una sanción penal y esta varía dependiendo de los meses de gestación que lleva el embarazo (arts. 118 y119).

Este es un tema de gran controversia en la población costarricense, una encuesta realizada por el Instituto de Estudios en Población (IDESPO) en el 2015, muestra que casi un 59\% de la población manifestaba estar de acuerdo con la realización del aborto terapéutico, sin embargo, este porcentaje se reducía a casi un $35 \%$ cuando se consultaba sobre la posibilidad de realizar un aborto cuando la mujer quedaba embarazada tras una violación (IDEsPo, 2015).
A pesar de la controversia en relación a este tema, solo cuatro partidos se pronuncian al respecto, de manera favorable lo hace el PT, mientras que indican oponerse el PRc, el PRN y el ML. Es necesario indicar que la oposición del mL se realiza de manera más sutil que la de los otros partidos, ya que abiertamente no expresa oponerse a la realización de abortos en Costa Rica, sino que hace un llamado a promover los métodos anticonceptivos como un medio para evitar los embarazos no deseados y por ende, los abortos (Partido Movimiento Libertario, 2013).

\section{LA AGENDA CONSERVADORA-RELIGIOSA EN LOS DEBATES TELEVISIVOS}

En este apartado se analizarán los discursos expresados por los cinco candidatos que mantuvieron una intención de voto mayor al margen de error reportado por las encuestas de opinión pública, durante la campaña electoral (Otto Guevara, José María Villalta, Rodolfo Piza, Luis Guillermo Solís y Jhonny Araya); y de los dos candidatos cuyos partidos políticos se reconocen abiertamente como cristianos o evangélicos (Justo Orozco y Carlos Avendaño). El propósito es observar cómo la agenda conservadora-religiosa se posiciona en las propuestas políticas de dichos candidatos y cómo es utilizada en su discurso para tratar de atraer los votos del electorado costarricense.

Como muestra la tabla 2, los temas más mencionados en los debates fueron el aborto $y$ la unión de personas del mismo sexo, seguido por el uso de valores cristianos para gobernar. En cuanto a los candidatos, quien hace mayor referencia a temas relacionados con la agenda conservadora durante los debates televisivos son Carlos Avendaño (PRN) y Otto Guevara (мL), seguidos por Justo Orozco (PRC). 


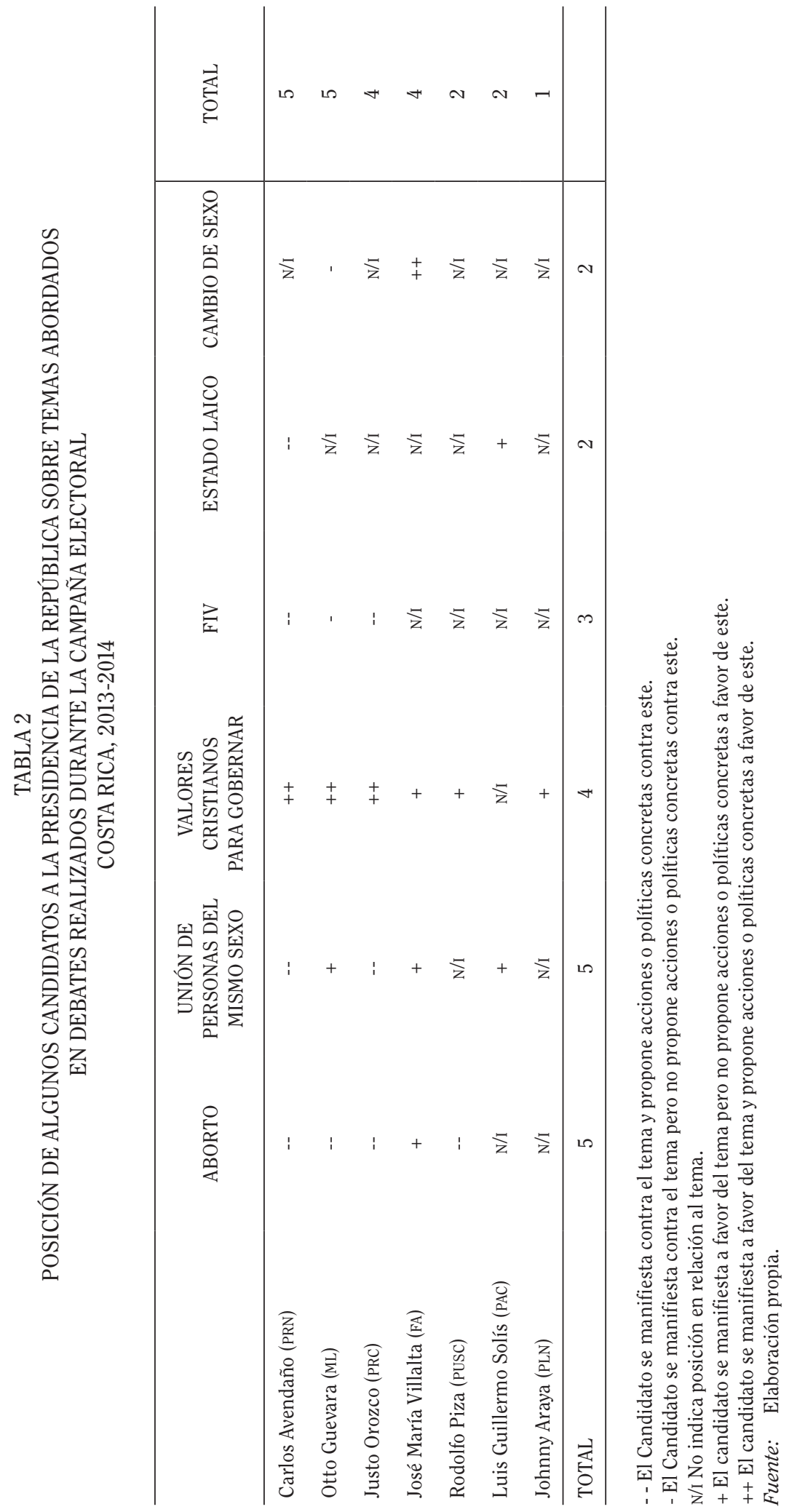


Respecto al tema del aborto, cuatro de los cinco candidatos que se pronuncian sobre este es para manifestarse en contra. Es necesario indicar que en muchos casos, no solo se manifiestan en contra del aborto, la legalización de las uniones del mismo sexo, riv, etc., debido a que sean contrarios a sus valores religiosos, sino también porque son temas que se vinculan con la izquierda. Al respecto, es necesario señalar que desde finales de 1930, se gesta en Costa Rica un fuerte movimiento "anti-comunista", el cual tiene su máxima expresión en la Constitución de 1949, en la cual se prohíbe de manera expresa la existencia de partidos políticos comunistas o de izquierda (Molina, 2007). Si bien, esta prohibición fue anulada en 1972, sigue en el imaginario político costarricense un fuerte rechazo a ideas o posiciones ideológicas de "izquierda". Así, esta situación se ve claramente reflejada en la siguiente manifestación realizada por Carlos Avendaño en el debate organizado por el IFED:

Quiero hacerle un llamado a todos los costarricenses de principios $y$ valores cristianos porque en estas elecciones se juega el destino de nuestro país, muchos quieren, o algunos quieren llevar hacia la izquierda a nuestro país, quieren no solamente un estado laico o ateo, que habría que discutir a ver que es la cosa, sino que también aprobar el aborto, aprobar el matrimonio homosexual, aprobar la fecundación in vitro...(Avendaño, 2014, el resaltado es propio).

Así, la narrativa generada por aquellos candidatos que se oponen a estos temas es la de señalar que son los partidos de izquierda quienes promueven temas contrarios a los "valores cristianos". Por ejemplo, en el mismo debate organizado por el IFED, Otto Guevara (мь) indica que los dos partidos de izquierda que participan en el proceso electoral (рт у ға) mantienen las mismas posiciones respecto a estos temas:

Don Héctor [pT], Don José María Villalta [FA] ha dicho que es ateo, que no cree en Dios. José María Villalta ha dicho que está a favor del aborto en cualquier circunstancia y ha dicho además que está a favor del matrimonio homosexual ¿En qué se diferencia usted de José María Villalta en esos tres temas? (Guevara, 2014a).

Incluso, en el debate organizado por la televisora Teletica (Canal 7), realizado el 19 de enero de 2014, Otto Guevara presiona a José María Villalta para que se vuelva a pronunciar sobre los temas del aborto en caso de violación, las uniones entre personas del mismo sexo y las operaciones de cambio de sexo; bajo el supuesto de que el fa presenta muchas ambigüedades y tiene que aclarar su posición sobre estos temas:

Don José María, ustedes en el Frente Amplio proponen que la Caja pague las operaciones de cambio de sexo, proponen el matrimonio homosexual con posibilidades de adopción y el aborto en caso de violación y malformaciones genéticas, como usted se ha estado contradiciendo tanto $y$ ha tenido tantas ambigüedades últimamente en relación a muchos temas, mantiene usted la posición de estar de acuerdo en esas tesis, diga sí o no en cada uno de esos casos, aborto por violación, matrimonio homosexual y operaciones de cambio de sexo (Guevara, 2014b).

Los ataques que se realizan a los partidos de izquierda que apoyan temas relacionados con los derechos sexuales y reproductivos, no solo se explican debido a las posturas o creencias religiosas de los candidatos que hacen dichos cuestionamientos; sino que también pueden tener una intención de minar el apoyo electoral de las agrupaciones de izquierda, especialmente del Fa, ya que una gran parte del electorado costarricense se manifiesta en contra de dichos temas. Por ejemplo, en una encuesta a nivel nacional realizada en el 2011 por el Instituto de Estudios Sociales en Población (IDESPO), se encontró que el 30\% de la población encuestada estaba en contra de que se realizará la fiv en Costa Rica. Otra encuesta realizada por el mismo centro de investigación en el 2015, determinó que el porcentaje de costarricenses que se oponían a la FIv aumentó a casi un 38\%. Respecto a la legalización de las uniones de personas del mismo 
sexo, este estudio encontró que un poco más del $51 \%$ de la población costarricense se encontraba en contra; asimismo, un $56 \%$ se oponía a la realización de abortos en caso de embarazo por violación (IDESPO, 2015).

Por lo tanto, la insistencia del mL, el PRN $y$ el prc de señalar que los "partidos de izquierda" promovían una agenda favorable al aborto, legalización de uniones del mismo sexo y fiv, respondía a una estrategia para tratar que el electorado no apoyara a dichas agrupaciones, esto especialmente en el caso del $\mathrm{FA}$, cuyo apoyo electoral durante la campaña 2013-2014 aumentó de tal manera, que muchas encuestas lo colocaban como la segunda agrupación con mayor intención de voto, detrás únicamente del PLN (ciep, 2014)

En lo que refiere al Estado laico, solo fue abordado por dos candidatos durante los debates; esto a pesar que fue uno de los temas con mayor impulso de la Iglesia Católica costarricense para ser colocado en la agenda electoral (Díaz, 2015). El primero es Carlos Avendaño, quien considera que un Estado laico es igual a un Estado ateo, al mismo tiempo que indica que si Costa Rica deja de ser un Estado confesional esto implicaría la desaparición de los partidos "cristianos". Estas declaraciones las realiza como un cuestionamiento hacia el pac, por unas supuestas declaraciones realizadas por Ana Elena Chacón, candidata a la Vicepresidencia de la República por dicha agrupación política:

Don Luis Guillermo, yo lo he escuchado a usted sobre el tema del Estado Laico, sobre el tema del aborto, sobre el tema de fecundación in vitro, de sociedades de convivencia o matrimonio homosexual, pero los diputados del pac son los principales impulsadores de esos proyectos en la Asamblea Legislativa y también dice Doña María Elena Chacón, su vicepresidenta en el Semanario Universidad, Ana Elena, que para que se respeten los derechos humanos en Costa Rica deben desaparecer el Estado confesional, osea tenemos que tener un estado ateo $y$ por otro lado dice que también deben desaparecer los partidos cristianos (...)
¿Qué es un Estado Laico? Porque para algunos es un Estado Ateo y no un Estado Laico, eso lo planteo como una pregunta, pero lo que ella dijo, es muy claro, de que los partidos cristianos o como lo dice ella religiosos tienen que desaparecer para que se respeten los derechos humanos aquí en Costa Rica (Avendaño, 2014).

Ante este cuestionamiento, Luis Guillermo Solís aclara que el promover un Estado laico no significa que buscan que el Estado sea ateo, al mismo tiempo, señala que tampoco desean proscribir a ningún partido político:

De hecho lo que tenemos que ir es hacer es una democracia más plural don Carlos, en donde no sea el fundamentalismo religioso de ningún tipo el que prevalezca, si no la oportunidad de todas $y$ todos de expresar su punto de vista (...)

Don Carlos no confunda un Estado Laico con un Estado Ateo, eso no lo dice ni siquiera las organizaciones religiosas y mucho menos la Iglesia Católica, un Estado Laico pero un Estado con Dios es el que quiere el Partido Acción Ciudadana (Solís, 2014).

Este intercambio entre candidatos en los debates, así como, lo reseñado hasta el momento en el presente artículo, es ilustrativo de como se usaron los elementos y temas relacionados con la religión y la agenda conservadora en la campaña electoral 2013-2014 en Costa Rica. Estos son utilizados para atacar a las agrupaciones políticas y candidatos que proponen cambios respecto a estos, pero sin entrar a discutir los motivos de fondo o las razones por los que deben conservarse o cambiarse. Por lo tanto, lo que se busca es crear ante el electorado la idea de que ciertos partidos se ligan o promueven temas controversiales o que no tienen amplio apoyo ante la opinión pública.

Por último, se encuentra el tema del "uso de valores cristianos para gobernar", el cual es utilizado en el discurso de todos los candidatos 
analizados, excepto en el de Luis Guillermo Solís. En este aspecto, resalta como José María Villalta utiliza la lectura de encíclicas del Papa Francisco durante el debate, para demostrar que su política social e ideología es congruente con las enseñanzas de la Iglesia Católica (Villalta, 2014), lo cual puede interpretarse como una táctica para apartarse de las etiquetas de ateo $y$ anti-religioso que otros partidos buscan ponerle. En otras palabras, sería una estrategia para tratar de combatir los ataques que otras agrupaciones políticas les hacen por su posición ante temas de la agenda conservadora-religiosa.

\section{CONCLUSIONES}

Al revisar el contenido de los planes de gobierno de los partidos políticos que contendían a la Presidencia de la República de Costa Rica para las elecciones del 2014, se encuentra que muchos de estos hacen eco de temas relacionados a la agenda conservadora-religiosa presente en el país. Sin embargo, aquellos que se posicionan a favor de dicha agenda lo hacen de manera tímida. Asimismo, varias agrupaciones políticas proponen acciones o políticas contrarias a esta agenda. Por lo tanto, al menos en lo que respecta a sus planes formales de gobierno, no parece haber excesivo interés de los partidos políticos a favor de la agenda conservadora-religiosa, en formular políticas o exponer sus ideas para su defensa y promoción. Al contrario, los partidos que tienen propuestas para defender o ampliar los derechos sexuales $y$ reproductivos, tienden a exponer de forma más clara y precisa sus intenciones y acciones.

En cuanto al discurso expresado durante los debates realizados por los candidatos con mayor intención de voto y aquellos provenientes de partidos cristianos, se encuentra que la mención a temas relacionados con la agenda conservadora-religiosa parece ser baja. Asimismo, cuando se recurre a estos temas, los candidatos a favor de esta agenda no lo hacen para defender su posición, sino para atacar a los candidatos opositores y especialmente, mostrar que la oposición a estos temas es un asunto de la "izquierda".

Sobre este aspecto debe señalarse que el candidato que recurre a un menor uso de elementos relacionados con la agenda religiosaconservadora es Luis Guillermo Solís; de hecho, en los debates hace referencia a estos de manera "defensiva", cuando es increpado por otros candidatos sobre la posición del PAC o de algunos de sus miembros sobre el Estado laico o el aborto. Al respecto, Carballo (2015 y 2016) ha determinado que Solís trató de adoptar durante los debates una postura de equilibrio o "centro", lo cual lo distanció de las controversias y conflictos generados por las posturas de los restantes candidatos. Dado esto, la falta de mención y posicionamiento en temas relacionados con la agenda religiosa-conservadora puede entenderse como parte de su estrategia discursiva de consolidarse como la opción de "centro político".

Por lo tanto, se encuentra un cambio interesante en el manejo del discurso en relación a los temas de la agenda conservadora-religiosa: por escrito (programas de gobierno) aquellas agrupaciones que se oponen a dicha agenda están más anuentes de dejar clara su oposición y los partidos políticos que promueven esta agenda lo hacen de manera vaga o incluso, omiten mencionarla. De manera oral (debates televisivos) los papeles cambian.

Como se indicó al inicio del artículo, para el 2013 un 73\% de la población costarricense se declaraba católica, $15 \%$ se declaraba de afiliación religiosa evangélica y solo un $8 \%$ indicaba no tener religión (cIEP, 2013); si bien, estos datos no permiten inferir que tan importante es la religión para la población que declara tener algún tipo de afiliación religiosa cristiana o si son practicantes regulares de su religión, permite constatar la importancia que mantiene en la sociedad costarricense, aunque sea solo de manera simbólica o nominal.

Dado lo anterior, no es de extrañar que los temas vinculados con la religión y la agenda religiosa-conservadora tengan presencia en la campaña electoral costarricense 2013-2014. Sin embargo, como se ha mostrado en el artículo, estos no tienen una posición central en el discurso político. Esto no quiere decir que no tenga influencia sobre los y las votantes (algo que es imposible de determinar en este texto), sino que no fue la base del discurso políticoelectoral manifestado por la mayoría de los 
aspirantes a la Presidencia de la República; más bien fue utilizado como una estrategia discursiva para atacar la posición de otros candidatos y partidos políticos.

Steigenga (2001) ha mostrado que, para finales del siglo xx, la disposición del electorado costarricense hacia la religión no parece ser un predictor confiable de su comportamiento electoral; no obstante, este autor señala que había un proceso de apropiación de elementos provenientes del pentecostalismo en el discurso de la clase política costarricense. Al respecto, dada la naturaleza de los datos analizados, no es posible valorar con certeza si la primera apreciación señalada sobre el comportamiento electoral de la población costarricense ha variado; sin embargo, sobre el segundo punto, los datos obtenidos al revisar el discurso electoral en relación a los temas de la agenda religiosa-conservadora lleva a pensar que esta situación no solo se ha consolidado, sino que incluso puede ser cada vez más importante para algunos partidos el incorporar elementos religiosos en su discurso político.

Así, tras considerar lo expuesto hasta el momento, puede suponerse que el peso de la religión para determinar el comportamiento del electorado costarricense ha variado del escenario que existía a inicios del siglo xxi (al menos así lo estiman algunos grupos políticos). De ahí su interés de adoptar y defender temas provenientes de la agenda religiosa-conservadora, como una manera de captar el apoyo de un cierto sector de la ciudadanía. Dada esta situación, es necesario empezar a incorporar las variables relacionadas con las creencias y la disposición de la población respecto a la religión, en los estudios de comportamiento electoral a desarrollar en Costa Rica, para así poder visualizar de manera más certera el peso que tienen estos temas en la definición de la intención del voto.

Por último, es necesario indicar dos limitaciones que presenta el análisis. La primera, que no hay estudios similares realizados sobre las campañas políticas anteriores en Costa Rica, por lo cual, no se puede establecer si la agenda religiosa-conservadora y el uso de elementos religiosos en el discurso manifestado por las personas aspirantes a la Presidencia de la República ha aumentado, disminuido o se ha mantenido. Una segunda limitación, es que no existen estudios sistemáticos sobre la implementación de políticas públicas provenientes de dicha agenda política-conservadora; por lo tanto, no es posible determinar si lo aludido por los partidos y los candidatos durante la campaña se queda solo en retórica, o si es probable que se implemente en caso de llegar a cargo del gobierno.

\section{REFERENCIAS}

Arguedas, G. (2010). El (aún) tortuoso camino hacia la emancipación: fundamentalismos religiosos, los derechos humanos de grupos históricamente oprimidos y la lucha por un Estado Laico en Costa Rica. Anuario del Centro de Investigación y Estudios Políticos (1), 50-65.

Avendaño, C. (2014). Debate con candidatos a la Presidencia de la República organizado por el Instituto en Formación y Estudios en Democracia.

Bastian, J-P. (1999). Los nuevos partidos políticos confesionales evangélicos $y$ su relación con el Estado en América Latina. Estudios Sociológicos xvir (49), 153-74.

Bastian, J-P. (2012). La mutación religiosa de América Latina: Para una sociología del cambio social en la modernidad periférica. México DF: Fondo de Cultura Económica.

Bastian, J-P. (2013). Protestantismos y modernidad latinoamericana. México DF: Fondo de Cultura Económica.

Carballo, P. (2015). Debatir o no debatir: he ahí la cuestión. Una reflexión de la campaña electoral en Costa Rica, 2013-2014. Revista de Ciencias Sociales I (147), 27-48.

Carballo, P. (2016). Tendencias, fracturas y quiebres en la discursividad políticoelectoral costarricense. Análisis políticodiscursivo durante el proceso de la campaña presidencial 2013-2014. Revista de Ciencias Sociales III (153), 147-71.

Casimigilia, G. y Tusón, A. (1999). Las cosas del decir. Barcelona: Ariel.

Centro de Investigación en Estudio Poblacionales [CIEP]. (2013). Estudio de 
Opinión Sociopolítica, agosto 2013”. Centro de Investigación y Estudios Políticos, Universidad de Costa Rica (UCR). Recuperado de http://www.ciep.ucr. ac.cr/index.php/publicaciones/estudiosopinion/Encuesta-de-Opini\%C3\%B3nAgosto-del-2013/

Centro de Investigación en Estudio Poblacionales [CIEP]. (2014). Estudio de Opinión Sociopolítica, enero 2014. Centro de Investigación y Estudios Políticos, Universidad de Costa Rica (UCR). Recuperado de http://www.ciep.ucr. ac.cr/index.php/publicaciones/estudiosopinion/Encuesta-de-Opini\%C3\%B3nEnero-del-2014/

Díaz, J. A. (2016). El discurso del periódico La Nación sobre la participación política de las personas jóvenes en Costa Rica (20092014). Revista Rupturas 6 (2), 301-28.

Díaz, J.A. (2012). Prensa escrita costarricense ante el referéndum sobre uniones de personas del mismo sexo (2010). Revista Rupturas 2 (2), 294-326.

Díaz, J.A. (2015). El discurso del Eco Católico en el proceso electoral costarricenses, 2013-2014. presentado en el VII Congreso Centroamericano de Ciencias Politicas, Universidad de Costa Rica, Costa Rica.

Editor (22 de junio de 2012). PAse cambia discurso y dice que apoyará proyecto de ley de unión entre parejas del mismo sexo. El Periodicocr.com. Recuperado de http://elperiodicocr.com/pase-cambiadiscurso-y-dice-que-apoyara-proyectode-ley-de-union-entre-parejas-delmismo-sexo/

Freston, P. (2001). Evangelicals and Politics in Asia, Africa and Latin America. Cambridge: Cambridge University Press.

Fuentes, L. (2006). Las apuestas del poder sobre el cuerpo de las mujeres. Las relaciones entre el Estado, la Iglesia Católica y el movimiento feminista alrededor del aborto en Nicaragua y Costa Rica. Anuario de Estudios Centroamericanos (32), 97-138.

Guevara, O. (2014a). Debate con candidatos a la Presidencia de la República organizado por el Instituto en Formación y Estudios en Democracia.

Guevara, O. (2014b). Debate con candidatos a la Presidencia de la República organizado por Teletica.

Instituto de Estudios Sociales en Población [IDESPO]. (2015). Percepción sobre aspectos de la coyuntura y las culturas políticas en Costa Rica. Universidad Nacional de Costa Rica.

Martín, S. (2009). Historiografía lingüistica $y$ análisis del discurso: las relaciones necesarias. Revista Argentina de Historiografía Lingüística I (1), 50-66.

Molina, I. (2007). Anticomunismo reformista: competencia electoral y cuestión social en Costa Rica (1931-1948). San José: Editorial Costa Rica.

Murillo, Á. (16 de enero de 2014). Cristianismo, el nuevo giro en el discurso de Otto Guevara. La Nación. Recuperado de http://www.nacion.com/nacional/ elecciones2014/Cristianismo-nuevo-girodiscurso-Guevara_0_1392860783.html

Nicolás, Y.; Alvarado, E.; Rodríguez, J. y Abarca, A. (2015). Las promesas electorales de los candidatos a la presidencia de la República de Costa Rica, divulgadas en medios de prensa escrita, 2014. Revista de Ciencias Sociales I (147), 17-26.

Partido Movimiento Libertario (2013). Las propuestas de Otto Guevara. Recuperado de http://www.archivoelectoral.org/ documentos/las-propuestas-de-otto/850

Partido Renovación Costarricense (2013). Plan de Gobierno. Recuperado de http://electoral.estadonacion.or.cr/files/ prc/PRC_propuesta2014.pdf.

Partido Restauración Nacional (2013). Por la restauración de una Costa Rica Solidaria. Nuestros lineamientos programáticos (2014-2018). Recuperado de http://www. tse.go.cr/aplicacionvisualizador/partidos/ programasPoliticos/2014/33.pdf

Partido Unidad Social Cristiana (2013). Programa de Gobierno: El camino costarricense, 2014-2018. Recuperado de https://issuu.com/rodolfopiza/docs/ plan_de_gobierno. 
Programa Umbral Político (2015). Percepción sobre aspectos de la coyuntura y las culturas politicas en Costa Rica. Instituto de Estudios Sociales en Población, Universidad Nacional, Costa Rica (una).

Quesada, C. (2012). Familia y Heteronormatividad: Acontecimientos históricos y la doctrina sexual de la Iglesia Católica en Costa Rica. Anuario de Estudios Centroamericanos 38, 305-28.

Rodríguez, F. (2013). tse declara parcialmente con lugar recurso contra partidos religiosos. Recuperado de http://www. ameliarueda.com/nota/tse-declaraparcialmente-con-lugar-recurso-contrapartidos-religiosos

Rodríguez, W. E. (22 de marzo de 2013). Partido Patria Nueva: Decálogo Ciudadano. Recuperado de http://partidopatrianueva. blogspot.com/2013/01/partido-patrianueva-costa-rica.html

Smith, B. (1998). Religious Politics in Latin America: Pentecostal vs. Catholic. Notre Dame: University of Notre Dame.

Solís, L. G. (2014). Debate con candidatos a la Presidencia de la República organizado por el Instituto en Formación y Estudios en Democracia.
Steigenga, T. (2001). The Politics of the Spirit: The Political Implications of Pentecostalized Religion in Costa Rica and Guatemala. Lanham: Lexinton Books.

Van Dijk, T. (2000). El discurso como interacción de la sociedad. En Van Dijk, T. (ed.) El discurso como interacción social. Estudios sobre el discurso II: Una introducción multidisciplinaria. Barcelona: Gedisa, s.A.

Vargas, L. P. (2013). La diversifobia como cultura, discurso y práctica constitutiva en la institucionalidad pública de Costa Rica. Revista Rupturas 1 (3), 44-87.

Villalta, J. M. (2014). Debate con candidatos a la Presidencia de la República organizado por Teletica.

Wodak, R. y Meyer, M. (2003). Métodos de análisis crítico del discurso. Barcelona: Gedisa, s.A.

Woshinsky, O. (2008). Explaining Politics. Culture, Institutions, and Political Behavior. $1^{\text {a }}$. New York: Routledge.

Fecha de ingreso: 25/01/2017 Fecha de aprobación: 21/04/2017 
